\title{
THE UNREASONABLE EFFECTUALNESS OF CONTINUED FUNCTION EXPANSIONS
}

\author{
GREG MARTIN
}

(Received 22 July 2002; revised 20 August 2003)

Communicated by W. W. L. Chen

\begin{abstract}
Many generalizations of continued fractions, where the reciprocal function has been replaced by a more general function, have been studied, and it is often asked whether such generalized expansions can have nice properties. For instance, we might ask that algebraic numbers of a given degree have periodic expansions, just as quadratic irrationals have periodic continued fractions; or we might ask that familiar transcendental constants such as $e$ or $\pi$ have periodic or terminating expansions. In this paper, we show that there exist such generalized continued function expansions with essentially any desired behaviour.
\end{abstract}

2000 Mathematics subject classification: primary 11J70; secondary 40A15.

\section{Introduction}

The familiar continued fraction expansion of a real number has great importance in its approximation by rational numbers, and the predictable behaviour of the continued fractions of certain classes of real numbers has added benefits. For example, the fact that the continued fraction expansion of a rational number terminates is essentially a re-expression of the Euclidean algorithm; also, the periodicity of the continued fractions for quadratic irrationals is crucial for calculating the fundamental units of real quadratic fields. Already in $1848 \mathrm{Hermite}$, in correspondence with Jacobi, asked about the existence of generalizations of continued fractions such that algebraic numbers of given degree would have periodic expansions. Since that time, myriad different generalizations have been studied (see [2] for an extensive list). Herein we focus on the $f$-expansions introduced by Bissinger [1], which we define momentarily. The purpose of this paper is to demonstrate that the function $f$ can be chosen so that the

(C) 2004 Australian Mathematical Society 1446-7887/04 \$A2.00+0.00 
expansions of prescribed real numbers can have essentially any desired behaviour. The following results, listed in roughly increasing order of unlikeliness, are representative of what we can prove.

THEOREM 1. For any two real numbers $x, y \in(0,1)$, there exists a function $f$ such that the $f$-expansion of $x$ is the same as the usual continued fraction expansion of $y$.

THEOREM 2. There exists a function $f$ such that the $f$-expansion of any rational or quadratic irrational terminates.

THEOREM 3. There exists a function $f$ such that the $f$-expansion of a real number $x$ is periodic if and only if $x$ is a cubic irrational number.

THEOREM 4. There exists a function $f$ such that, simultaneously for every integer $d \geq 1$, a real number $x$ is algebraic of degree $d$ if and only if the $f$-expansion of $x$ terminates with the integer $d+1$.

We remark that Voronoì's algorithm [4] for calculating units in number fields of degree higher than two is not directly relevant to Theorem 3 , since we are interested in generalizations that give well-defined expansions for every real number. We also remark that in all four theorems, the cardinality of the set of functions $f$ satisfying the given property is that of the continuum, which is the cardinality of the set of all continuous functions on the real numbers. Finally, we mention an even more surprising generalization of Theorem 4 , which we discuss in more detail later in the paper: there exists a function $f$ such that the $f$-expansion of every algebraic real number $x$ of degree $d$ terminates with the integer $d+1$, and the $d+1$ integers directly preceding this final $d+1$ encode the minimal polynomial of $x$.

Let us describe more precisely the class of expansions we shall consider. The output of any such expansion will be a sequence in the set $\mathscr{C}=\mathscr{C}_{i} \cup \mathscr{C}_{t}$, where

$$
\mathscr{C}_{i}=\left\{\left[a_{0} ; a_{1}, a_{2}, \ldots\right]: \text { each } a_{j} \in \mathbb{Z}, a_{j} \geq 1 \text { for all } j \geq 1\right\}
$$

and

$$
\begin{aligned}
\mathscr{C}_{t}= & \left\{\left[a_{0} ; a_{1}, \ldots, a_{n}\right]: n \geq 0, \text { each } a_{j} \in \mathbb{Z},\right. \\
& \left.a_{j} \geq 1 \text { for all } 1 \leq j \leq n, a_{n} \geq 2 \text { if } n \geq 1\right\} .
\end{aligned}
$$

We emphasize that the elements of these sets are formal sequences of integers, not real numbers; the sets $\mathscr{C}_{i}$ and $\mathscr{C}_{t}$ are the infinite and terminating sequences, respectively. Let $\lfloor x\rfloor$ and $\{x\}=x-\lfloor x\rfloor$ denote the greatest integer function and fractional part, respectively, of $x$. Define $\mathscr{F}$ to be the set of decreasing homeomorphisms from $(1, \infty)$ to $(0,1)$, that is, the set of all strictly decreasing continuous functions $f$ defined on $(1, \infty)$ satisfying $\lim _{x \rightarrow 1^{+}} f(x)=1$ and $\lim _{x \rightarrow+\infty} f(x)=0$. Throughout this paper, 
$f$ will denote a function from the class $\mathscr{F}$ and $\phi$ will denote the inverse of $f$, so that $\phi$ is a decreasing homeomorphism from $(0,1)$ to $(1, \infty)$.

We define the expansion function $E_{f}: \mathbb{R} \rightarrow \mathscr{C}$ (sometimes called the representation function by other authors) as follows. Given $x_{0} \in \mathbb{R}$, we set $a_{0}=\left\lfloor x_{0}\right\rfloor$. If $x_{0}$ is not an integer, we set $x_{1}=\phi\left(\left\{x_{0}\right\}\right)$ and $a_{1}=\left\lfloor x_{1}\right\rfloor$. If $x_{1}$ is not an integer, we set $x_{2}=\phi\left(\left\{x_{1}\right\}\right)$ and $a_{2}=\left\lfloor x_{2}\right\rfloor$, and so on. Then the value of $E_{f}\left(x_{0}\right)$ is $\left[a_{0} ; a_{1}, \ldots, a_{n}\right] \in \mathscr{C}$, or $\left[a_{0} ; a_{1}, a_{2}, \ldots\right] \in \mathscr{C}_{i}$, depending on whether one of the $x_{j}$ is equal to an integer. The prototypical example uses the reciprocal function $r(x)=1 / x$, in which case $E_{r}\left(x_{0}\right)$ is the usual continued fraction expansion of $x_{0}$. In general, we call $E_{f}\left(x_{0}\right)$ the continued function expansion of $x_{0}$, or sometimes the continued $f$-expansion for a specific function $f$. In the terminology of [2], these are $f$-expansions of type $\mathrm{A}$ $(f$-expansions of type B are formed from increasing functions $f$ and generalize the usual decimal expansions of real numbers).

In this paper, when we write simply $\left[a_{0} ; a_{1}, a_{2}, \ldots\right]$ we mean the formal element of $\mathscr{C}$. If we want to refer to the real number whose usual continued fraction expansion is $\left[a_{0} ; a_{1}, a_{2}, \ldots\right]$, we use the notation $\left[a_{0} ; a_{1}, a_{2}, \ldots\right]$. In general, for any function $f \in \mathscr{F}$ we define an evaluation function $V_{f}$ on $C_{t}$ recursively by setting $V_{f}\left(\left[a_{0}\right]\right)=a_{0}$ and $V_{f}\left(\left[a_{0} ; a_{1}, \ldots, a_{n}\right]\right)=a_{0}+f\left(V_{f}\left(\left[a_{1} ; a_{2}, \ldots, a_{n}\right]\right)\right)$. Thus

$$
V_{f}\left(\left[a_{0} ; a_{1}, \ldots, a_{n}\right]\right)=a_{0}+f\left(a_{1}+f\left(a_{2}+\cdots+f\left(a_{n}\right) \cdots\right)\right),
$$

which is the continued $f$-expansion of a certain real number. We extend the definition of $V_{f}$ to as much of $\mathscr{C}$ as we can by defining

$$
V_{f}\left(\left[a_{0} ; a_{1}, a_{2}, \ldots\right]\right)=\lim _{n \rightarrow \infty} V_{f}\left(\left[a_{0} ; a_{1}, \ldots, a_{n}\right]\right)
$$

when the limit exists. We shall often write $\left[a_{0} ; a_{1}, \ldots, a_{n}\right]_{f}$ as a shorthand for $V_{f}\left(\left[a_{0} ; a_{1}, \ldots, a_{n}\right]\right)$, thus generalizing the notation $\left[a_{0} ; a_{1}, a_{2}, \ldots\right]_{r}$ given above.

Note that $\left[a_{0} ; a_{1}, a_{2}, \ldots\right]$, always exists and equals the unique real number $x$ such that $E_{r}(x)=\left[a_{0} ; a_{1}, a_{2}, \ldots\right]$, that is, the expansion function $E_{r}: \mathbb{R} \rightarrow \mathscr{C}$ and the evaluation function $V_{r}: \mathscr{C} \rightarrow \mathbb{R}$ are inverses of each other. For a general function $f \in \mathscr{F}$, the definitions of $E_{f}$ and $V_{f}$ do imply that the restriction of $V_{f}$ to $\mathscr{C}_{t}$ and the restriction of $E_{f}$ to $V_{f}\left(\mathscr{C}_{t}\right)$ are inverses of each other; however, $E_{f}$ might not be injective on all of $\mathbb{R}$, or $V_{f}$ might not be defined on all of $\mathscr{C}$, and so on.

If the function $f \in \mathscr{F}$ does have the property that $E_{f}$ and $V_{f}$ are inverses of each other, we call $f$ a faithful function. In other words, $f$ is faithful if and only if $E_{f}$ is bijective and the limit defining $\left[a_{0} ; a_{1}, a_{2}, \ldots\right]_{f}$ exists for every element of $C_{i}$ and always equals the unique real number $x$ such that $E_{f}(x)=\left[a_{0} ; a_{1}, a_{2}, \ldots\right]$. (The list of properties in this last sentence is probably redundant for characterizing faithful functions, though we shall not need a more streamlined criterion. Our definition of faithful is related to what other authors call a valid representation.) In this terminology, 
Theorems $1-4$ can be stated more precisely using the phrase 'there exists a faithful function $f \in \mathscr{F}$ such that the continued $f$-expansion $E_{f}$ of ....'

The idea of our method is to endow $\mathscr{C}$ with a topology that is naturally related to continued fraction expansions and then to treat the expansion and evaluation maps $E_{f}$ and $V_{f}$ as continuous functions between $\mathbb{R}$ and $\mathscr{C}$. We describe this topology and begin to explore its consequences in Section 2. With this foundation, we can make substantial progress by composing these functions with carefully chosen continuous functions from $\mathbb{R}$ to itself. This technique, which leads to proofs of Theorems 14 , is expounded in Section 3. Finally, in Section 4 we investigate the class of continued function expansions given by power functions $f(x)=x^{-\alpha}$, including several numerical examples that partially motivated this paper.

At this point we should confess what the reader might already suspect, that the functions giving the nice behaviours of Theorems 1-4 are infeasible for actual computations. Indeed, the existence of such functions is essentially a consequence of the existence of continuous functions on the interval $(0,1)$ with certain properties. We have chosen the title of this paper, a subtle variation on the famous phrase 'unreasonable effectiveness' pioneered by Wigner [5], for this reason. Mirriam-Webster's Collegiate Dictionary contrasts the two words by saying that 'effective' in fact 'stresses the actual production of or the power to produce an effect', while 'effectual' merely 'suggests the accomplishment of a desired result especially as viewed after the fact'. We cannot think of a more apt description of these techniques.

\section{Topological preliminaries}

We recall that if $S$ is a set endowed with a total linear ordering, the order topology on $\mathrm{S}$ is defined by declaring the open sets to be arbitrary unions of open intervals in $S$, that is, of sets of the form $(x, y)=\{s \in S: x<s<y\}$. If $S$ and $T$ are two ordered sets, a function $f: S \rightarrow T$ is order preserving if, whenever $x<y$ in $S$, we have $f(x)<f(y)$ in $T$. It is easily checked that this implies $x<_{S} y \Leftrightarrow f(x)<_{T} f(y)$, and consequently any order-preserving function is automatically injective. We shall need the following result as well:

LEMMA 5. Let $h: S \rightarrow T$ be a function between the two ordered spaces $S$ and $T$. If $h$ is order-preserving and surjective, then $h$ is a homeomorphism.

PROOF. Since any order-preserving function is injective, $h$ is in fact a bijection, and it is easily verified that $h^{-1}$ is also order-preserving. Moreover, it is true that the image under $h$ of any open interval $(x, y) \subset S$ is exactly the open interval $(h(x), h(y))$ in $T$ : certainly the image is contained in this open interval by the order-preserving property of $h$, while every point in $(h(x), h(y))$ must have a preimage in $S$ by the surjectivity 
of $h$, and this preimage must be in $(x, y)$ again by order-preservation. This shows that both $h$ and $h^{-1}$ are continuous, and therefore $h$ is a homeomorphism.

We can endow $\mathscr{C}$ with the alternating lexicographic order topology, or the alt-lex topology for short, which is the order topology defined by the following total ordering of $\mathscr{C}$ :

$$
\begin{aligned}
{\left[a_{0} ; a_{1}, a_{2}, \ldots\right] } & <\left[b_{0} ; b_{1}, b_{2}, \ldots\right] \\
& \Longleftrightarrow\left(a_{0}<b_{0}\right) \text { or }\left(a_{0}=b_{0} \text { and } a_{1}>b_{1}\right) \\
& \text { or }\left(a_{0}=b_{0} \text { and } a_{1}=b_{1} \text { and } a_{2}<b_{2}\right) \\
& \text { or }\left(a_{0}=b_{0} \text { and } a_{1}=b_{1} \text { and } a_{2}=b_{2} \text { and } a_{3}>b_{3}\right) \text { or } \ldots
\end{aligned}
$$

Terminating elements $\left[a_{0} ; a_{1}, \ldots, a_{n}\right] \in \mathscr{C}_{1}$ are treated as $\left[a_{0} ; a_{1}, \ldots, a_{n},+\infty\right]$ when applying this definition. It is easy to see that for any $f \in \mathscr{F}$, the evaluation map $E_{f}$ is semi-order-preserving, that is, $x \leq y$ in $\mathbb{R}$ implies that $E_{f}(x) \leq E_{f}(y)$ in $\mathscr{C}$. In particular, if $f$ is a faithful function, then the function $E_{f}$ is bijective and hence strictly order preserving. We conclude from Lemma 5 that the evaluation function $E_{f}$ of any faithful $f \in \mathscr{F}$ is a homeomorphism from $\mathbb{R}$ to $\mathscr{C}$.

Lemma 6. A subset $B$ of $\mathscr{C}$ is dense if and only if, for every element $x=$ $\left[a_{0} ; a_{1}, \ldots, a_{n}\right]$ of $\mathscr{C}_{t}$, there exists an element $b=\left[b_{0} ; b_{1}, b_{2}, \ldots\right]$ of $B$ such that $b_{0}=a_{0}, b_{1}=a_{1}, \ldots, b_{n}=a_{n}$.

PROOF. The key observation is that the set of elements $x=\left[x_{0} ; x_{1}, x_{2}, \ldots\right]$ of $C$ such that $x_{0}=a_{0}, x_{1}=a_{1}, \ldots, x_{n}=a_{n}$ is one of the half-open intervals $\left(\left[a_{0} ; a_{1}, \ldots, a_{n}+1\right],\left[a_{0} ; a_{1}, \ldots, a_{n}\right]\right]$ or $\left[\left[a_{0} ; a_{1}, \ldots, a_{n}\right],\left[a_{0} ; a_{1}, \ldots, a_{n}+1\right]\right)$, depending on whether $n$ is odd or even. Every half-open interval of this form obviously contains an open interval, which proves the 'only if' part of the lemma; conversely, every open interval $(c, d)$ in $C$ contains a half-open interval of this form (let $n-1$ be the first index at which the elements $c$ and $d$ differ), which proves the 'if' part of the lemma.

PROPOSITION 7. Let $A$ and $B$ be two countable dense subsets of $(0,1)$. Then there exists an increasing homeomorphism $g:(0,1) \rightarrow(0,1)$ such that $g(A)=B$.

Proof. Note that any dense subset of $(0,1)$ must in fact be infinite. Fix any wellorderings of $A$ and $B$ (that is, arrange the elements of $A$ and $B$ into infinite sequences); we emphasize that this well-ordering is not related to the orderings of $A$ and $B$ as subsets of $(0,1)$. We recursively construct a sequence of order-preserving bijections $g_{j}: A_{j} \rightarrow B_{j}$, where $A_{j}$ and $B_{j}$ are subsets of $A$ and $B$, respectively, as follows. 
Choose any elements $a_{1} \in A$ and $b_{1} \in B$, and define $A_{1}=\left\{a_{1}\right\}, B_{1}=\left\{b_{1}\right\}$, and $g_{1}\left(a_{1}\right)=b_{1}$.

If $n \geq 2$ is even, we extend $A_{n-1}, B_{n-1}$, and $g_{n-1}$ as follows. Choose the first (in the fixed well-ordering) element $b \in B \backslash B_{n-1}$. If $b$ is smaller than every element of $B_{n-1}$, choose $a \in A \backslash A_{n-1}$ that is smaller than every element of $A_{n-1}$. If $b$ is larger than every element of $B_{n-1}$, choose $a \in A \backslash A_{n-1}$ that is larger than every element of $A_{n-1}$. If neither of these cases holds, then there are unique elements $c, d$ of the finite set $B_{n-1}$ such that $c<b<d$ and $(c, d) \cap B_{n-1}=\emptyset$; choose $a \in A \backslash A_{n-1}$ such that $g_{n-1}^{-1}(c)<a<g_{n-1}^{-1}(d)$. (All of these choices are possible since $A$ is dense in $(0,1)$.) After making this choice, we set $A_{n}=A_{n-1} \cup\{a\}$ and $B_{n}=B_{n-1} \cup\{b\}$, and we define $g_{n}: A_{n} \rightarrow B_{n}$ by $g_{n}(a)=b$ and $g_{n}(x)=g_{n-1}(x)$ if $x \in A_{n-1}$.

Similarly, if $n \geq 3$ is odd, we extend $A_{n-1}, B_{n-1}$, and $g_{n-1}$ as follows. Choose the first (in the fixed well-ordering) element $a \in A \backslash A_{n-1}$. If $a$ is smaller than every element of $A_{n-1}$, choose $b \in B \backslash B_{n-1}$ that is smaller than every element of $B_{n-1}$. If $a$ is larger than every element of $A_{n-1}$, choose $b \in B \backslash B_{n-1}$ that is larger than every element of $B_{n-1}$. If neither of these cases holds, then there are unique elements $c, d$ of the finite set $A_{n-1}$ such that $c<a<d$ and $(c, d) \cap A_{n-1}=\emptyset$; choose $b \in B \backslash B_{n-1}$ such that $g_{n-1}(c)<b<g_{n-1}(d)$. (All of these choices are possible since $B$ is dense in $(0,1)$.) After making this choice, we set $A_{n}=A_{n-1} \cup\{a\}$ and $B_{n}=B_{n-1} \cup\{b\}$, and we define $g_{n}: A_{n} \rightarrow B_{n}$ by $g_{n}(a)=b$ and $g_{n}(x)=g_{n-1}(x)$ if $x \in A_{n-1}$.

It is easy to verify inductively that each $g_{n}$ is a bijection from $A_{n}$ to $B_{n}$ that is order-preserving with respect to the usual order on $(0,1)$, and that $\left.g_{n}\right|_{A_{m}}=g_{m}$ for all positive integers $m<n$. Furthermore, the use of the well-orderings of $A$ and $B$ during the construction forces $\bigcup_{n \geq 1} A_{n}=A$ and $\bigcup_{n \geq 1} B_{n}=B$. Therefore, there is a unique function $g_{\infty}: A \rightarrow B$ (namely the union of all the functions $g_{n}$ ) such that $\left.g_{\infty}\right|_{A_{n}}=g_{n}$ for all $n \geq 1$, and in fact $g_{\infty}$ is an order-preserving bijection from $A$ to $B$.

Finally, define $g:(0,1) \rightarrow(0,1)$ by $g(x)=\sup \left\{g_{\infty}(a): a \in A, a<x\right\}$ for $x \in(0,1)$. Note that for any $x \in(0,1)$, there exist $a_{1}, a_{2} \in A$ such that $a_{1}<x<a_{2}$ (by the density of $A$ ), whence the set $\left\{g_{\infty}(a): a \in A, a<x\right\}$ is bounded above by $g_{\infty}\left(a_{1}\right)<1$ and contains $g_{\infty}\left(a_{2}\right)>0$ by the order-preservation of $g_{\infty}$; therefore $g(x)$ is a well-defined real number in $(0,1)$. Also, $g$ is order preserving: if $c, d \in(0,1)$ with $c<d$, then there exists $a_{1}, a_{2} \in A$ with $c<a_{1}<a_{2}<d$, whence

$$
\begin{aligned}
g(c) & =\sup \left\{g_{\infty}(a): a \in A, a<c\right\} \leq g_{\infty}\left(a_{1}\right) \\
& <g_{\infty}\left(a_{2}\right) \leq \sup \left\{g_{\infty}(a): a \in A, a<d\right\}=g(d) .
\end{aligned}
$$

Moreover, $g$ is surjective: given $y \in(0,1)$, define $x=\sup \left\{g_{\infty}^{-1}(b): b \in B, b<y\right\}$. Because $g_{\infty}$ is an order-preserving bijection from $A$ to $B$ and $B$ is dense near $y$, it is easy to check that $g(x)=y$. Therefore $g$ is an order-preserving surjection from $(0,1)$ to $(0,1)$, hence a homeomorphism by Lemma 5 . 
A straightforward extension of this construction yields the following:

PROPOSITION 8. Let $A_{1}, A_{2}, \ldots$ be a collection (finite or countably infinite) of pairwise disjoint countable dense subsets of $(0,1)$, and similarly for $B_{1}, B_{2}, \ldots$ Then there exists an increasing homeomorphism $g:(0,1) \rightarrow(0,1)$ such that $g\left(A_{1}\right)=B_{1}$, $g\left(A_{2}\right)=B_{2}$, and so on.

\section{Proofs of the theorems}

We call a function $h: \mathbb{R} \rightarrow \mathbb{R}$ a chorus-line function if $h$ maps the set $(0,1)$ into itself and $h(x)=\lfloor x\rfloor+h(\{x\})$ for all real numbers $x$. This definition implies that $\lfloor h(x)\rfloor=\lfloor x\rfloor$ and $\{h(x)\}=h(\{x\})$ for all $x$. Let $\mathscr{G}$ denote the set of increasing homeomorphisms $g:(0,1) \rightarrow(0,1)$. To any $g \in \mathscr{G}$ we may associate a function $\bar{g}: \mathbb{R} \rightarrow \mathbb{R}$, called the chorus-line extension of $g$, defined by

$$
\bar{g}(x)= \begin{cases}x, & \text { if } x \text { is an integer; } \\ \lfloor x\rfloor+g(\{x\}), & \text { if } x \text { is not an integer. }\end{cases}
$$

It is easy to see that $\bar{g}$ is an increasing homeomorphism from $\mathbb{R}$ to itself and that both $\bar{g}$ and $\bar{g}^{-1}=\overline{g^{-1}}$ are chorus-line functions. For any $g \in \mathscr{G}$, we define $f_{g}$ to be the restriction of the function $g^{-1}$ or $\circ \bar{g}$ to the domain $(1, \infty)$, so that $f_{g}(x)=g^{-1}(1 / \bar{g}(x))$ for $x>1$. It is again easy to see that $f_{g} \in \mathscr{F}$ with inverse $\phi_{g}=\bar{g}^{-1} \circ r \circ g$.

PROPOSITION 9. Let $g \in G$. Then $f_{g}$ is a faithful function satisfying

$$
\bar{g}\left(\left[a_{0} ; a_{1}, a_{2}, \ldots\right]_{f_{g}}\right)=\left[a_{0} ; a_{1}, a_{2}, \ldots\right]_{r}
$$

for every $\left[a_{0} ; a_{1}, a_{2}, \ldots\right] \in \mathscr{C}$. In other words, the continued $f$-expansion of every real number $x$ is identical to the usual continued fraction expansion of $\bar{g}(x)$.

PROOF. Let $x \in \mathbb{R}$, and let $E_{r}(\bar{g}(x))=\left[a_{0} ; a_{1}, a_{2}, \ldots\right]$; we want to show as a first step that $E_{f_{s}}(x)=\left[a_{0} ; a_{1}, a_{2}, \ldots\right]$ as well. For each $n \geq 0$, define $y_{n}=$ $\left[a_{n} ; a_{n+1}, a_{n+2}, \ldots\right]_{r}$ and $x_{n}=\bar{g}^{-1}\left(y_{n}\right)$, so that $y_{0}=\bar{g}(x)$ and $x_{0}=x$. Notice that

$$
\left\lfloor x_{n}\right\rfloor=\left\lfloor\bar{g}^{-1}\left(y_{n}\right)\right\rfloor=\left\lfloor y_{n}\right\rfloor=a_{n}
$$

for all $n \geq 0$, where we have used the fact that $\bar{g}^{-1}$ is a chorus-line function. Similarly,

$$
\begin{aligned}
\phi_{g}\left(\left\{x_{n}\right\}\right) & =\bar{g}^{-1} \circ r \circ g\left(\left\{\bar{g}^{-1}\left(y_{n}\right)\right\}\right) \\
& =\bar{g}^{-1} \circ r \circ g\left(\bar{g}^{-1}\left(\left\{y_{n}\right\}\right)\right) \\
& =\bar{g}^{-1} \circ r\left(\left\{y_{n}\right\}\right)=\bar{g}^{-1}\left(y_{n+1}\right)=x_{n+1} .
\end{aligned}
$$


Now, simply considering the definition of the evaluation function $E_{f_{g}}\left(x_{0}\right)$ in light of equations (2) and (3) reveals that $E_{f_{g}}(x)=E_{f_{g}}\left(x_{0}\right)=\left[a_{0} ; a_{1}, a_{2}, \ldots\right]$ as desired.

This shows that $E_{f_{s}}=E_{r} \circ \bar{g}$; in particular, $E_{f_{s}}$ is the composition of two homeomorphisms and is therefore itself a homeomorphism, with $E_{f_{g}}^{-1}=\bar{g}^{-1} \circ E_{r}^{-1}$. To show that $f_{g}$ is faithful, it only remains to show that $V_{f_{g}}$ is well-defined on all of $\mathscr{C}$ and inverts the function $E_{f_{g}}$. As above, let $x \in \mathbb{R}$ and $E_{f_{z}}(x)=E_{r}(\bar{g}(x))=\left[a_{0} ; a_{1}, a_{2}, \ldots\right]$; we want to show that $V_{f_{g}}\left(\left[a_{0} ; a_{1}, a_{2}, \ldots\right]\right)=\lim _{n \rightarrow \infty} V_{f_{g}}\left(\left[a_{0} ; a_{1}, \ldots, a_{n}\right]\right)$ exists and equals $x$. Now $V_{f_{s}}$ does invert $E_{f_{s}}$ on $\mathscr{C}_{t}$, so

$$
\begin{aligned}
V_{f_{g}}\left(\left[a_{0} ; a_{1}, \ldots, a_{n}\right]\right) & =E_{f_{g}}^{-1}\left(\left[a_{0} ; a_{1}, \ldots, a_{n}\right]\right) \\
& =\bar{g}^{-1} \circ E_{r}^{-1}\left(\left[a_{0} ; a_{1}, \ldots, a_{n}\right]\right) \\
& =\bar{g}^{-1} \circ V_{r}\left(\left[a_{0} ; a_{1}, \ldots, a_{n}\right]\right)=\bar{g}^{-1}\left(\left[a_{0} ; a_{1}, \ldots, a_{n}\right]_{r}\right) .
\end{aligned}
$$

However, we know that $\lim _{n \rightarrow \infty}\left[a_{0} ; a_{1}, \ldots, a_{n}\right]_{r}=\left[a_{0} ; a_{1}, a_{2}, \ldots\right]_{r}$ by the convergence of usual continued fractions. Therefore, by the continuity of $\bar{g}^{-1}$, we have

$$
\begin{aligned}
V_{f_{s}}\left(\left[a_{0} ; a_{1}, a_{2}, \ldots\right]\right) & =\lim _{n \rightarrow \infty} \bar{g}^{-1}\left(\left[a_{0} ; a_{1}, \ldots, a_{n}\right]_{r}\right) \\
& =\bar{g}^{-1}\left(\left[a_{0} ; a_{1}, a_{2}, \ldots\right]_{r}\right)=\bar{g}^{-1}(\bar{g}(x))=x
\end{aligned}
$$

as desired.

Together, Propositions 7-9 imply each of Theorems 1-4. For example, given $x, y \in(0,1)$, let $g \in \mathscr{G}$ be chosen so that $g(x)=y$. Then Proposition 9 tells us that $f_{g}$ is a faithful function in $\mathscr{F}$ and that $E_{f_{g}}(x)=E_{r}(y)$, which is precisely the statement of Theorem 1 . In fact, $g$ can be chosen to be piecewise linear, in which case $f_{g}$ is given piecewise by Möbius transformations $(a x+b) /(c x+d)$.

For any positive integer $d$, let $Q(d)$ denote the set of numbers in $(0,1)$ that are algebraic over $\mathbb{Q}$ of degree exactly $d$, so that $Q(1)=\mathbb{Q} \cap(0,1)$ for instance. Each $Q(d)$ is a countable dense subset of $(0,1)$. Therefore, Proposition 7 tells us that there exists a function $g \in \mathscr{G}$ such that $g(Q(3))=Q(2)$. We then know from Proposition 9 that the corresponding $f_{g}$ has the property that the continued $f_{g}$-expansion of any number in $Q(3)$ is the same as the usual continued fraction expansion of a number in $Q(2)$, and vice versa. Since the usual continued fraction expansion of a real number is periodic if and only if the number is a quadratic irrational, this $f_{g}$ gives a faithful function such that the continued $f_{\mathrm{g}}$-expansion of a real number $x$ is periodic if and only if $x$ is a cubic irrational number, establishing Theorem 3 .

A similar approach establishes Theorem 2. In fact, the singular Minkowski function $?(x)$ (see [3]) is an increasing homeomorphism of $(0,1)$ that was constructed to have the property that $?(Q(1) \cup Q(2))=Q(1)$. Therefore $f_{?} \in \mathscr{F}$ is a faithful function such that the continued $f_{\text {? }}$-expansion of any rational or quadratic irrational number terminates. 
As for Theorem 4 , we partition $\mathscr{C}_{t}$ into infinitely many sets $\mathscr{C}_{t}(1), \mathscr{C}_{t}(2), \ldots$, where we define

$$
\begin{aligned}
\mathscr{C}_{\imath}(d)= & \left\{\left[0 ; a_{1}, \ldots, a_{n}\right]: n \geq 1, \text { each } a_{j} \in \mathbb{Z},\right. \\
& \left.a_{j} \geq 1 \text { for all } 1 \leq j<n, a_{n}=d+1\right\} .
\end{aligned}
$$

Each $\mathscr{C}_{t}(d)$ is dense in $\mathscr{C}$ by Lemma 6. Therefore, if we define $Q(1, d)=V_{r}\left(\mathscr{C}_{t}(d)\right)$, then $Q(1,1), Q(1,2), \ldots$ is a partition of $\mathbb{Q} \cap(0,1)$ into countably many countably infinite subsets, each dense in $(0,1)$. Applying Proposition 8 , we can find a function $g \in \mathscr{G}$ such that $g(Q(d))=Q(1, d)$ for every positive integer $d$. Then, by Proposition 9 , the set of real numbers in $(0,1)$ whose continued $f_{g}$-expansion terminates in the integer $d+1$ is precisely $Q(d)$.

We briefly discuss the extension of Theorem 4 mentioned in the introduction. We generalize the notation of the previous paragraph by defining $\mathscr{C}_{t}\left(n_{1}, n_{2}, \ldots, n_{k}\right)$ to be the set of terminating expansions in $\mathscr{C}$ that begin with a zero and end with the $k$ integers $n_{1}, \ldots, n_{k}$, and we set $Q\left(1, n_{1}, \ldots, n_{k}\right)=V_{r}\left(\mathscr{C}_{t}\left(n_{1}, n_{2}, \ldots, n_{k}\right)\right)$. We choose a function that encodes every integer, positive or negative, as a positive integer; one such function is

$$
\eta(k)= \begin{cases}2|k|+1, & \text { if } k \leq 0 \\ 2 k, & \text { if } k>0\end{cases}
$$

which is a bijection from $\mathbb{Z}$ to $\mathbb{Z}^{+}$whose inverse is $\delta(k)=(-1)^{k}\lfloor k / 2\rfloor$. Using a modification of Proposition 8 , we can find a function $g \in \mathscr{G}$ such that, for each algebraic number $x \in(0,1)$, if the minimal polynomial of $x$ is $c_{d} t^{d}+c_{d-1} t^{d-1}+\cdots+$ $c_{1} t+c_{0}$, then $g(x) \in \mathscr{C}_{t}\left(\eta\left(c_{0}\right), \eta\left(c_{1}\right), \ldots, \eta\left(c_{d-1}\right), \eta\left(c_{d}\right), d+1\right)$. (Many transcendental numbers would also be mapped into these subsets of $\mathscr{C}_{t}$ by $g$.) The corresponding faithful function $f_{g} \in \mathscr{F}$ would then have the property that the continued $f_{g}$-expansion of $x$ would terminate in the sequence $\eta\left(c_{0}\right), \eta\left(c_{1}\right), \ldots, \eta\left(c_{d-1}\right), \eta\left(c_{d}\right), d+1$, thus encoding the minimal polynomial of $x$. If we had an oracle that could compute this function $f_{g}$ quickly, we could test whether any real number $y$ was algebraic by computing its continued $f_{\mathrm{g}}$-expansion; if it terminated, say as $\left[0 ; a_{1}, \ldots, a_{n}\right]$, then $y$ would be either transcendental or else a root of the polynomial

$$
\delta\left(a_{n-1}\right) t^{a_{n}-1}+\delta\left(a_{n-2}\right) t^{a_{n}-2}+\cdots+\delta\left(a_{n-\left(a_{n}-1\right)}\right) t+\delta\left(a_{n-a_{n}}\right) .
$$

Of course, this is only a fantasy, as the function $f_{g}$ is hopelessly infeasible for exact computation. Other types of encoding functions are possible, of course; for example, one can encode every finite sequence of integers as a single positive integer via some Gödel-type code.

Proposition 9 shows us how we can contruct a faithful function in $\mathscr{F}$ from any function in $\mathscr{G}$. The following result demonstrates that the opposite is also true: 
PROPOSITION 10. Let $f \in \mathscr{F}$ be a faithful function. Define $g$ to be the restriction of the function $V_{r} \circ E_{f}$ to $(0,1)$. Then $g \in \mathscr{G}$ and $f_{g}=f$.

Proof. Since $E_{f}:(0,1) \rightarrow \mathscr{C}$ and $V_{r}: \mathscr{C} \rightarrow(0,1)$ are both order preserving, we see from Lemma 5 that $g$ is indeed a homeomorphism from $(0,1)$ to itself, and hence $g \in \mathscr{G}$. Notice that $V_{r} \circ E_{f}$ has the property that $V_{r} \circ E_{f}(x)=\lfloor x\rfloor+V_{r} \circ E_{f}(\{x\})$; therefore $V_{r} \circ E_{f}$ is its own chorus-line extension and so $\bar{g}=V_{r} \circ E_{f}$. If we write $E_{f}(x)=\left[a_{0} ; a_{1}, a_{2}, \ldots\right]$, then $\bar{g}(x)=V_{r}\left(\left[a_{0} ; a_{1}, a_{2}, \ldots\right]\right)=\left[a_{0} ; a_{1}, a_{2}, \ldots\right]_{r}$ and therefore $r \circ \bar{g}(x)=\left[0 ; a_{0}, a_{1}, a_{2}, \ldots\right]_{r}$. Finally, note that $\left(V_{r} \circ E_{f}\right)^{-1}=E_{f}^{-1} \circ V_{r}^{-1}=$ $V_{f} \circ E_{r}$, and so

$$
\begin{aligned}
g^{-1} \circ r \circ \bar{g}(x) & =V_{f} \circ E_{r}\left(\left[0 ; a_{0}, a_{1}, a_{2}, \ldots\right]_{r}\right)=\left[0 ; a_{0}, a_{1}, a_{2}, \ldots\right]_{f} \\
& =0+f\left(\left[a_{0} ; a_{1}, a_{2}, \ldots\right]_{f}\right)=f(x)
\end{aligned}
$$

as desired.

We say that two functions $f, f^{\prime} \in \mathscr{F}$ are chorus-line conjugate if there exists an increasing continuous chorus-line function $h$ such that $f$ equals the restriction of $h^{-1} \circ f^{\prime} \circ h$ to the domain $(1, \infty)$. It is easy to check that chorus-line conjugacy is an equivalence relation on $\mathscr{F}$. The next theorem shows that the equivalence class containing the reciprocal function $r$ is precisely the class of faithful functions.

THEOREM 11. Let $f \in \mathscr{F}$. The following are equivalent:

(i) $f$ is faithful;

(ii) $f$ is chorus-line conjugate to $r$;

(iii) $f=f_{g}$ for some $g \in \mathscr{G}$.

In particular, there is a one-to-one correspondence between functions $g \in \mathscr{G}$ and faithful functions $f \in \mathscr{F}$.

PROOF. It is easy to see that any increasing continuous chorus-line function is equal to $\bar{g}$ for some $g \in \mathscr{G}$. Thus $f$ and $r$ are chorus-line conjugate if and only if $f=\bar{g}^{-1} \circ r \circ \bar{g}=f_{g}$ for some $g \in \mathscr{G}$, which shows the equivalence of statements (ii) and (iii). Proposition 9 shows that statement (iii) implies statement (i), while Proposition 10 shows that statement (i) implies statement (iii). Therefore the three statements are indeed equivalent.

The assertion that there is a one-to-one correspondence between functions $g \in \mathscr{G}$ and faithful functions $f \in \mathscr{F}$ requires some justification, as it is not immediately clear that different functions $g, g^{\prime} \in \mathscr{G}$ give rise to distinct $f_{\mathrm{g}}$ and $f_{g^{\prime}}$. Suppose that $g \neq g^{\prime}$, and choose an $x \in(0,1)$ such that $g(x) \neq g^{\prime}(x)$. By Proposition 9, we have $E_{f_{g}}(x)=E_{r}(g(x)) \neq E_{r}\left(g^{\prime}(x)\right)=E_{f_{g^{\prime}}}(x)$. Since the continued $f$ - and $f^{\prime}$-expansions of $x$ differ, we must have $f_{g} \neq f_{g^{\prime}}$. 
At the end of the last proof, we used the fact that two faithful functions whose corresponding expansion functions are different must be distinct. In fact, the converse is also true:

PROPOSITION 12. Suppose that $f_{1}, f_{2} \in \mathscr{F}$ are faithful functions with the property that $E_{f_{1}}=E_{f_{2}}$. Then $f_{1}=f_{2}$.

Proof. Given $x \in(1, \infty)$, we want to prove that $f_{1}(x)=f_{2}(x)$. Write $E_{f_{1}}(x)=$ $\left[a_{0} ; a_{1}, a_{2}, \ldots\right]=E_{f_{2}}(x)$. Note that the continued $f_{1}$-expansion of $f_{1}(x)=0+f_{1}(x)$ is $\left[0 ; a_{0}, a_{1}, a_{2}, \ldots\right]$, so that $E_{f_{1}}\left(f_{1}(x)\right)=\left[0 ; a_{0}, a_{1}, a_{2}, \ldots\right]$. Similarly, $E_{f_{2}}\left(f_{2}(x)\right)=$ $\left[0 ; a_{0}, a_{1}, a_{2}, \ldots\right]$. But then $E_{f_{1}}\left(f_{2}(x)\right)=\left[0 ; a_{0}, a_{1}, a_{2}, \ldots\right]$ since $E_{f_{1}}=E_{f_{2}}$. Therefore $E_{f_{1}}\left(f_{1}(x)\right)=E_{f_{1}}\left(f_{2}(x)\right)$, and since $f_{1}$ is faithful, $E_{f_{1}}$ is injective and thus $f_{1}(x)=f_{2}(x)$.

\section{Continued power function expansions}

Let us consider a particular one-parameter family of functions from $\mathscr{F}$, namely the power functions $f_{\alpha}(x)=x^{-\alpha}$ for $\alpha>0$, so that $f_{1}=r$. A continued $f_{\alpha}$-expansion of a real number is thus an expression of the form

$$
a_{0}+\left(a_{1}+\left(a_{2}+\left(a_{3}+\left(a_{4}+\cdots\right)^{-\alpha}\right)^{-\alpha}\right)^{-\alpha}\right)^{-\alpha} .
$$

At a problem session of the West Coast Number Theory Conference in 1999, Kevin O'Bryant considered the case $\alpha=1 / 2$, which he called the continued root expansion of a real number $x$. For instance, some rational numbers such as

$$
\frac{2}{3}=0+\frac{1}{\sqrt{2+(1 / \sqrt{16})}}=[0 ; 2,16]_{f_{1 / 2}}
$$

and $27 / 47=[0 ; 3,1098,2892,410,256]_{f_{1 / 2}}$ have terminating continued root expansions. On the other hand, O'Bryant remarked that

$$
E_{f_{1 / 2}}(3 / 4)=[0 ; 1,1,2,8,5,1,3,3,14,321,2,300,1,13,2,6,1,1,2, \ldots]
$$

does not seem to terminate; but we do not know how to prove this. At the same problem session, Bart Goddard noted several other examples; for instance, $E_{f_{s}}(\sqrt[5]{7})=$ $[1 ; 1,1,1, \ldots]$, and the continued $f_{3 / 2}$-expansion of $\sqrt[3]{3}=1.44224957$ looks at first to be periodic of period four. However,

$$
\begin{aligned}
E_{f_{3 / 2}}(\sqrt[3]{3})= & {[1 ; 1,1,2,1,1,1,2,1,1,1,2,1,1,1} \\
& 3,1,1,1,1,3,1,2,1,1,7,23,1, \ldots]
\end{aligned}
$$


does not seem to be periodic, while

$$
x=V_{f_{3 / 2}}([1 ; 1,1,2,1,1,1,2,1,1,1,2, \ldots])=1.44225029
$$

is the nearby number that satisfies the equation

$$
x=1+\left(1+\left(1+\left(2+x^{-3 / 2}\right)^{-3 / 2}\right)^{-3 / 2}\right)^{-3 / 2} .
$$

In fact, writing $w, z$, and $y$ as the three quantities in parentheses on the right-hand side of this equation (starting from the innermost parentheses), we see that this number $x$ is the $x$-coordinate of one solution to the system of equations

$$
y^{3}(x-1)^{2}=1, \quad z^{3}(y-1)^{2}=1, \quad w^{3}(z-1)^{2}=1, \quad x^{3}(w-2)^{2}=1 .
$$

Using elimination theory and a computational algebra package, we can show that this number $x$ is algebraic of degree 93; more precisely, it is the fourth of seven real roots of the irreducible polynomial

$$
\begin{aligned}
- & 401+12348 x-22442 x^{2}+275800 x^{3}-1337555 x^{4}+2423872 x^{5} \\
& -15418480 x^{6}+70540444 x^{7}-127417629 x^{8}+557491285 x^{9} \\
& -2405709582 x^{10}+4329064154 x^{11}-14625356403 x^{12}+59525595995 x^{13} \\
& -106704972668 x^{14}+296336967716 x^{15}-1137325584809 x^{16} \\
& +2031978559593 x^{17}-4823156208926 x^{18}+17439240838410 x^{19} \\
& -31080157671439 x^{20}+64755935263191 x^{21}-220128009411364 x^{22} \\
& +391629168869836 x^{23}-730457802870121 x^{24}+2326819690217101 x^{25} \\
& -4133151272936538 x^{26}+7008134858873413 x^{27}-20830642337065947 x^{28} \\
& +36915446021983793 x^{29}-57610938763130172 x^{30} \\
& +159042225095378801 x^{31}-280663409776128761 x^{32} \\
& +407135007678293093 x^{33}-1039187243118822998 x^{34} \\
& +1820679151953897429 x^{35}-2473096725871085456 x^{36} \\
& +5813652442623811749 x^{37}-10072679129493093706 x^{38} \\
+ & 12871647850762706121 x^{39}-27781471420314300292 x^{40} \\
& +47381905470113399929 x^{41}-57050802593263213282 x^{42} \\
& +112791554026161912586 x^{43}-188441383925133877380 x^{44} \\
+ & 213305384930045048629 x^{45}-385514084983483335018 x^{46} \\
+ & 627994700913994360904 x^{47}-663244567700776798728 x^{48} \\
+ & 1093132419527821059119 x^{49}-1729925336326989733586 x^{50}
\end{aligned}
$$




$$
\begin{aligned}
& +1677221045253645425438 x^{51}-2509661817013886167565 x^{52} \\
& +3855357757564686581031 x^{53}-3317198776437539175677 x^{54} \\
& +4459703033666698711524 x^{55}-6699690356527027205257 x^{56} \\
& +4712835619541841129045 x^{57}-5515895022295988234329 x^{58} \\
& +8418986026761406210570 x^{59}-3557493739786303419709 x^{60} \\
& +2967144052401077482669 x^{61}-6097230519934320899607 x^{62} \\
& -2428850299115623275704 x^{63}+4729361127147513235131 x^{64} \\
& -963376378662124156885 x^{65}+11586996393861391188069 x^{66} \\
& -14002890770158170207742 x^{67}+7817852618475056747791 x^{68} \\
& -16575420482318059675255 x^{69}+16734537138028434957244 x^{70} \\
& -7089221324585019485886 x^{71}+10933203152887989317941 x^{72} \\
& -8907308593248131984589 x^{73}-1468466124295786901110 x^{74} \\
& +1320989510532080943648 x^{75}-2515839920964633664993 x^{76} \\
& +8733255119045045834197 x^{77}-8648102018082906320368 x^{78} \\
& +7228474474951901475700 x^{79}-7991643882573751006683 x^{80} \\
& +6679169691105510026567 x^{81}-4448501164546530714930 x^{82} \\
& +3131234600047636654702 x^{83}-1971348622249197779737 x^{84} \\
& +953049356660824435629 x^{85}-363152194705059550764 x^{86} \\
& +72557323790158601616 x^{87}+29118868029709313904 x^{88} \\
& -24792096645669431805 x^{89}+4762766285696524504 x^{90} \\
& +768224935099977754 x^{91}-343187952655081548 x^{92} \\
& +28598996054590129 x^{93}
\end{aligned}
$$

Continued $f_{\alpha}$ expansions exhibit several interesting phenomena which merit further study. For example, let us consider whether the limit defining $[1 ; 1,1,1, \ldots]_{f_{\alpha}}$ converges. Computationally, we find that there is a threshhold number $\alpha_{0}=4.1410415 \ldots$ with the property that $[1 ; 1,1,1, \ldots]_{f_{\alpha}}$ converges for all $0<\alpha<\alpha_{0}$ (which we refer to as 'small $\alpha^{\prime}$ ) but diverges for all $\alpha>\alpha_{0}$ ('large $\alpha^{\prime}$ ). In fact, $\alpha_{0}$ is the unique positive solution of the equation $y^{y}=(y-1)^{y+1}$.

The behaviour of $E_{f_{\alpha}}$ as $\alpha$ passes through $\alpha_{0}$ experiences a classic bifurcation. For all positive $\alpha$, the function $(x-1)^{-1 / \alpha}$ has a unique fixed point between 1 and 2; however, this fixed point is repelling for small $\alpha$ but attracting for large $\alpha$. Therefore only a single real number has $[1 ; 1,1,1, \ldots]$ as its continued $f_{\alpha}$-expansion for small $\alpha$, and $V_{f_{\alpha}}[1 ; 1,1,1, \ldots]$ converges back to this real number. In contrast, there is a whole interval of real numbers having $[1 ; 1,1,1, \ldots]$ as their continued $f_{\alpha}$-expansions for large $\alpha$. For example, when $\alpha=5$, all real numbers in the 
interval $(1.06377,1.73411)$ have $[1 ; 1,1,1, \ldots]$ as their continued $f_{5}$-expansion. In particular, since $\sqrt[5]{7}=1.47577$, the example $E_{f_{s}}(\sqrt[5]{7})=[1 ; 1,1,1, \ldots]$ mentioned above is less significant than it seems.

Indeed, for large $\alpha$ the function $\left((x-1)^{-\alpha}-1\right)^{-\alpha}$ has three fixed points in the interval $(1,2)$, the central one being unstable and the outer two being stable. The evaluations $V_{f_{a}}[1 ; 1,1, \ldots, 1]$ oscillate back and forth between ever-decreasing neighborhoods of the two stable fixed points as the number of ones increases, and hence $V_{f_{u}}[1 ; 1,1,1, \ldots]$ does not converge for large $\alpha$. The two outer fixed points approach 1 from above and 2 from below, respectively, as $\alpha$ tends to infinity. We can conclude, for instance, that for every real number $x \in(1,2)$, there exists an $\alpha(x)$ such that, whenever $\alpha>\alpha(x)$, we have $E_{f_{\alpha}}(x)=[1 ; 1,1,1, \ldots]$. For example, we have $E_{f_{\alpha}}(\sqrt[5]{7})=[1 ; 1,1,1, \ldots]$ for all $\alpha>4.26159$.

The above discussion implies in particular that $f_{\alpha}$ is not faithful for large $\alpha$. On the other hand, it can be shown that no analogous bifurcation occurs for $[n ; n, n, n, \ldots]$ when $n \geq 2$ (the key equality now becomes $n^{y+1} y^{y}=(y-1)^{y+1}$ which has no positive solution). Computational evidence suggests that periodic sequences of longer period never undergo bifurcations either. We are thus led to conjecture that $f_{\alpha}$ is faithful for all $0<\alpha<\alpha_{0}$. (Kakeya's theorem (see [2, Section 8.3]) is only relevant when $\alpha<1$.) In particular, this conjecture would imply by Theorem 11 that the power function $x^{-\alpha}$ is chorus-line-conjugate to the reciprocal function on $(1, \infty)$ for $\alpha<\alpha_{0}$ but not for $\alpha>\alpha_{0}$, a curious state of affairs.

We can also use these functions to show that the analogue of Proposition 12 for non-faithful functions does not hold. Indeed, if $f \in F$ is any function agreeing with $f_{5}$ outside the interval $(1.06377,1.73411)$, then it is easy to see that $E_{f}=E_{f_{s}}$.

We mention one last phenomenon, where we fix a real number $x$ and consider the function from $\mathbb{R}$ to $\mathscr{C}$ that maps $\alpha$ to $E_{f_{a}}(x)$. Counterintuitively, this function is not an order-preserving function of $\alpha$. For example, when $x=1 / 2$, the function $\alpha \mapsto E_{f_{\alpha}}(1 / 2)$ is increasing for $\alpha<2.24228$ but decreasing thereafter (stabilizing eventually at $[1 ; 1,1,1, \ldots]$, as we have already seen).

\section{Acknowledgements}

The author acknowledges the support of the Natural Sciences and Engineering Research Council and of the Department of Mathematics of the University of British Columbia, and thanks Bart Goddard for the interesting questions.

\section{References}

[1] B. H. Bissinger, 'A generalization of continued fractions', Bull. Amer. Math. Soc. 50(1944), 868-876. 
[2] F. Schweiger, Ergodic theory of fibred systems and metric number theory (The Clarendon Press Oxford University Press, New York, 1995).

[3] P. Viader, J. Paradís and L. Bibiloni, 'A new light on Minkowski’s ? $(x)$ function', J. Number Theory 73 (1998), 212-227.

[4] G. F. Voronoil, On a generalization of the algorithm of continued fractions (Ph.D. Thesis, Warsaw, 1896), (Russian).

[5] E. P. Wigner, 'The unreasonable effectiveness of mathematics in the natural sciences [Comm. Pure Appl. Math. 13 (1960), 1-14]', Mathematical analysis of physical systems (Van Nostrand Reinhold, New York, 1985) pp. 1-14.

Department of Mathematics

University of British Columbia

Room 121, 1984 Mathematics Road

Vancouver, BC V6T 1Z2

Canada

e-mail: gerg@math.ubc.ca 
